\title{
Comparación de la Maduración Ósea de Vértebras Cervicales Utilizando los Métodos de Baccetti y Lamparski en Pacientes de 8 a 15 Años
}

\author{
Comparison of Bone Maturation of Cervical Vertebrae Using Baccetti \\ and Lamparski Methods in Patients 8 to 15 Years of Age
}

Emerik Alvarado-Torres*; Jaime Fabián Gutiérrez-Rojo** \& Alma Rosa Rojas-García**

\begin{abstract}
ALVARADO-TORRES, E.; GUTIÉRREZ-ROJO, J. F. \& ROJAS-GARCÍA, A. R. Comparación de la maduración ósea de vértebras cervicales utilizando los métodos de Baccetti y Lamparski en pacientes de 8 a 15 años. Int. J. Odontostomat., 10(1):63-67, 2016.

RESUMEN: La maduración se puede definir como un proceso gradual en el tiempo, presenta sucesivas modificaciones cualitativas en la organización anatómica y fisiológica durante la pubertad hasta el estado de madurez adulto. En el diagnóstico y tratamiento en ortodoncia es importante conocer los estadios de maduración ósea pues proporcionan información relevante que permite determinar ritmo y tiempo de maduración individual. El uso y la aplicación varían entre las técnicas y tienen un objetivo común, la clasificación de niños y adolescentes en estadios de maduración. El objetivo de este estudio fue comparar la maduración ósea de las vértebras cervicales utilizando los métodos de Baccetti y Lamparski en una población nayarita. La muestra fueron 298 radiografías laterales de cráneo de las edades de 8 a 15 años. Se compararon mediante t de Student los estadios de maduración de los grupos en el análisis CVM de Baccetti y el análisis de Lamparski; en hombres y mujeres. La población masculina de 8 y 10 años no presentaba diferencias significativas entre ambos análisis; la población femenina de 8 años y masculina de 9 años se encontraron diferencias, teniendo mayores valores el análisis CVM de Baccetti, en los demás grupos del estudio también se encontraron diferencias presentando mayores valores en el análisis Lamparski. Los estadios de maduración en los dos tipos de análisis mostraron una marcada diferencia en sus interpretaciones, siendo el análisis de Lamparski más confiable como método diagnóstico de la maduración ósea.
\end{abstract}

PALABRAS CLAVE: maduración ósea, vértebras, Lamparski, Baccetti.

\section{INTRODUCCIÓN}

La maduración biológica es un proceso de importantes cambios fisiológicos que se manifiestan de forma más intensa durante la adolescencia y el tiempo de su aparición depende del sexo y la etapa de maduración. La maduración se puede definir como un proceso gradual en el tiempo, en el que se presentan sucesivas modificaciones cualitativas en la organización anatómica y fisiológica, a través del cual se desencadena una gran movilización hormonal durante la pubertad hasta alcanzar el estado de madurez adulto (Gómez-Campos et al., 2013).
En el diagnóstico y tratamiento en ortodoncia es importante conocer los estadios de maduración ósea (Mejía-Garduño et al., 2014), ya que en las personas de la misma edad cronológica existen muchas diferencias fisiológicas en su desarrollo, por lo que es necesario evaluar mediante un indicador de maduración ósea su desarrollo (Echarri, 1998).

Dichos estadios proporcionan información relevante que permite determinar el ritmo o tiempo de maduración individual. El uso y la aplicación varía entre

Estudiante de la Especialidad en Ortodoncia, Universidad Autónoma de Nayarit, Nayarit, México.

* Maestro en Salud Pública. Docente de la Especialidad en Ortodoncia y de la Unidad Académica de Odontología, Universidad Autónoma de Nayarit, Nayarit, México.

*** Maestra en Odontología. Docente de la Especialidad en Ortodoncia y de la Unidad Académica de Odontología, Universidad Autónoma de Nayarit, Nayarit, México. 
las técnicas, sin embargo, todos tienen un objetivo común, la clasificación de los niños y adolescentes en estadios de maduración (Gómez-Campos et al.).

San-Román et al. (2002), mencionan que el mejor indicador de maduración ósea de la morfología de las vértebras cervicales es la concavidad que se forma en la parte inferior del cuerpo de la vértebra cervical.

El uso de las vértebras cervicales como método para valorar la maduración ósea en radiografías cefálicas laterales fue empleado inicialmente en 1972 por Lamparski; en un estudio desarrollado en la Escuela Medicina Dental de la Universidad de Pittsburg se estableció una clasificación basado en los cambios que se observan en las vértebras cervicales. La muestra empleada fue seleccionada del departamento de Ortodoncia, con un total de 141 pacientes, 72 niñas y 69 niños entre 10-15 años de edad, caucásicos, con una oclusión de Clase I mesomórfico y sin patología previa (Chen et al., 2004; Ortiz et al., 2007).

Lampaski identificó y utilizó características morfológicas que presentaban las vértebras en cada uno de los períodos estudiados, con esto consiguió crear un "Atlas descriptivo de radiografías estándares". Por otro lado, realizó radiografías de muñecas asignando la edad ósea a cada paciente mediante el método de Grewlich \& Pyle. Entre las conclusiones del trabajo arrojaron que este método es estadísticamente fiable, valida y es clínicamente tan útil como la valoración esquelética mediante la radiografía de la muñeca. Otro hallazgo fue que los indicadores de maduración vertebral de niños y niñas son los mismos, la diferencia es que en las niñas cada estado de desarrollo se observa más temprano (Ortiz et al.).

Baccetti et al. (2005), realizaron un análisis de la maduración vertebral cervical (CVM) método basado en el análisis de la segunda a cuarta vértebras cervicales en un solo cefalograma. Fueron observadas la morfología de los órganos de la segunda (C2), tercera (C3) y cuarta (C4) vértebras cervicales en individuos sin tratamiento de ortodoncia.

El método CVM se compone de seis etapas de maduración (fase cervical 1 a través del cuello a la etapa 6, es decir, a través de CS1-CS6). CS1 y CS2 son etapas prepico; el pico en el crecimiento mandibular ocurre entre CS3 y CS4. CS6 se registra por lo menos 2 años después del pico. El uso del método CVM permite identificar el momento óptimo para el tratamiento de una serie de desarmonías dentoesqueléticas en los tres planos del espacio (Baccetti et al.).

Las ventajas de determinar la maduración ósea con las vértebras cervicales es que se puede utilizar la radiografía lateral que se emplea en el diagnóstico en ortodoncia y no hay necesidad de tomar otra radiografia (Gandini et al., 2006), es fácil de utilizar y de reproducir los resultados (Perinetti et al., 2014).

\section{MATERIAL Y MÉTODO}

Se revisaron 720 expedientes de pacientes que acuden a la clínica de Posgrado de la Unidad Académica de Odontología de la Universidad Autónoma de Nayarit, la muestra fue de 298 radiografías pretratamiento de Ortodoncia y Ortopedia que cumplieron con los criterios de inclusión, se clasificaron por edades y género obteniendo grupos de 20 pacientes de cada género de las edades de 10 a 15 años. Para los grupos de 8 años fue de 11 hombres y 15 mujeres y en el grupo de 9 años fueron 11 hombres y 20 mujeres; esto debido a que no se encontró la población suficiente para ajustar la muestra a 20 casos.

Los criterios de inclusión consistieron en expedientes completos con historia clínica y radiografía lateral de cráneo que fueran visibles las vértebras en su totalidad y que al momento de la toma de la radiografía el paciente contara con la edad de 8 a 15 años. Los criterios de exclusión fueron radiografías dañadas o deterioradas o con anomalías en la anatomía vertebral.

Los materiales utilizados fueron 298 radiografías laterales de cráneo, un negatoscopio, regla milimétrica, cámara fotográfica réflex Nikon 3200, el programa Nemotec Dental Studio versión 11, la historia clínica física de cada paciente, Laptop y el programa Microsoft Excel 2011.

Se tomó fotografía digital de cada radiografía lateral de cráneo y se registraron sus datos personales en una hoja de Excel, se generó el expediente digital en el programa Nemotec Dental Studio ${ }^{\circledR}$ versión 11 para cada paciente y posteriormente se realizó el análisis vertebral de CVM; y el análisis de Lamparski, los resultados se registraron en la hoja de Excel donde se encontraban sus datos personales, para su posterior análisis estadístico. 


\section{RESULTADOS}

Hombres. En hombres a los 8 años se presentó una media en el estadio de maduración CVM de 2,36 y en el análisis de Lamparski una media de 2,0, a los 9 años en el análisis CVM se obtuvo una media de 3,55 y en Lamparski de 2,45; a los 10 años de edad se encontró una media de maduración en CVM de 2,95 y en la Lamparski de 3,0.

A los 11 años se presentó una media en el estadio de maduración en el análisis CVM de 2,25 mientras que en Lamparski se obtuvo una media de 4,15, a los 12 años de edad se obtuvo una media en análisis CVM de 2,7 y en Lamparski mantuvo una media de 4,3 , en la edad de 13 años se obtuvo una media en la maduración en CVM de 2,4 y en el análisis de Lamparski de 4,9.

A los 14 años se presentó una media en CVM de 2,5 y en Lamparski de 5,2 y a los 15 años la media del análisis CVM fue de 2,7 y en Lamparski de 5,5, el resto de la estadística descriptiva se encuentra en las Tablas I y II.

Tabla I. Población Masculina en Análisis CVM de Baccetti.

\begin{tabular}{lccccc}
\hline Edad & Media & DE & Máximo & Mínimo & Rango \\
\hline 8 & 2,3 & 0,48 & 3,0 & 2,0 & 1,0 \\
9 & 3,5 & 1,0 & 5,0 & 1,0 & 4,0 \\
10 & 2,9 & 0,73 & 4,0 & 2,0 & 2,0 \\
11 & 2,5 & 0,62 & 4,0 & 1,0 & 3,0 \\
12 & 2,7 & 0,55 & 4,0 & 2,0 & 2,0 \\
13 & 2,4 & 0,73 & 4,0 & 1,0 & 3,0 \\
14 & 2,5 & 0,59 & 4,0 & 2,0 & 2,0 \\
15 & 2,7 & 0,53 & 4,0 & 2,0 & 2,0 \\
\hline
\end{tabular}

Tabla II. Población Masculina en Análisis de Lamparski.

\begin{tabular}{lccccc}
\hline Edad & Media & DE & Máximo & Mínimo & Rango \\
\hline 8 & 2,0 & 1,35 & 6,0 & 1,0 & 5,0 \\
9 & 2,4 & 0,9 & 4,0 & 1,0 & 3,0 \\
10 & 3,0 & 1,32 & 5,0 & 1,0 & 4,0 \\
11 & 4,1 & 1,65 & 6,0 & 1,0 & 5,0 \\
12 & 4,3 & 1,34 & 6,0 & 1,0 & 5,0 \\
13 & 4,9 & 1,39 & 6,0 & 2,0 & 4,0 \\
14 & 5,2 & 0,94 & 6,0 & 3,0 & 3,0 \\
15 & 5,5 & 0,66 & 6,0 & 4,0 & 2,0 \\
\hline
\end{tabular}

Mujeres. En mujeres a los 8 años se presentó una media en el estadio de maduración CVM de 3,6 y en el análisis de Lamparski una media de 2,2, a los 9 años en el análisis CVM se obtuvo una media de 2,8 y en Lamparski de 3,5; a los 10 años de edad se encon- tró una media de maduración en CVM de 2,6 y en la Lamparski de 3,9.

A los 11 años se presentó una media en el estadio de maduración en el análisis CVM de 2.7 mientras que en Lamparski se obtuvo una media de 4,5, a los 12 años de edad se obtuvo una media en análisis CVM de 2,3 y en Lamparski mantuvo una media de 5,0 , en la edad de 13 años se obtuvo una media en la maduración en CVM de 2,4 y en el análisis de Lamparski de 5,2.

A los 14 años se presentó una media en CVM de 2,4 y en Lamparski de 5,5 y a los 15 años la media del análisis CVM fue de 2,8 y en Lamparski de 5,2, el resto de la estadística descriptiva se encuentra en las Tablas III y IV.

Tabla III. Población Femenina en Análisis CVM de Bacceti.

\begin{tabular}{lccccc}
\hline Edad & Media & DE & Máximo & Mínimo & Rango \\
\hline 8 & 3,6 & 0,87 & 5,0 & 2,0 & 3,0 \\
9 & 2,8 & 0,67 & 4,0 & 2,0 & 2,0 \\
10 & 2,6 & 0,48 & 3,0 & 2,0 & 1,0 \\
11 & 2,7 & 0,76 & 4,0 & 1,0 & 3,0 \\
12 & 2,3 & 0,47 & 3,0 & 2,0 & 1,0 \\
13 & 2,4 & 0,66 & 3,0 & 1,0 & 2,0 \\
14 & 2,4 & 0,58 & 3,0 & 1,0 & 2,0 \\
15 & 2,8 & 0,60 & 4,0 & 2,0 & 2,0 \\
\hline
\end{tabular}

Tabla IV. Población Femenina en Análisis de Lamparski.

\begin{tabular}{lccccc}
\hline Edad & Media & DE & Máximo & Mínimo & Rango \\
\hline 8 & 2,2 & 1,06 & 4,0 & 1,0 & 3,0 \\
9 & 3,5 & 0,92 & 6,0 & 2,0 & 4,0 \\
10 & 3,9 & 1,33 & 5,0 & 1,0 & 4,0 \\
11 & 4,5 & 1,20 & 6,0 & 2,0 & 4,0 \\
12 & 5,0 & 1,16 & 6,0 & 2,0 & 4,0 \\
13 & 5,2 & 1,16 & 6,0 & 3,0 & 3,0 \\
14 & 5,5 & 0,97 & 6,0 & 2,0 & 4,0 \\
15 & 5,2 & 0,69 & 6,0 & 4,0 & 2,0 \\
\hline
\end{tabular}

Pruebas t. Se compararon mediante una prueba de $t$ de Student los estadios de maduración de los grupos de 8 años a 15 años en el análisis CVM de Baccetti con el análisis de Lamparski; en hombres como en mujeres. Se encontró que la población masculina de 8 y 10 años no presentaba diferencias estadísticamente significativas entre ambos análisis; sin embargo los grupos de población femenina de 8 años y masculina de 9 años se encontraron diferencias significativas, teniendo mayores valores el análisis CVM de Baccetti, en los demás grupos del estudio también se encontraron diferencias significativas (Tablas $\mathrm{V}$ y VI). 
Tabla V. Comparación de estadios de Análisis CVM de Baccetti y Análisis de Lamparski en grupos de edad en Hombres.

\begin{tabular}{lcc}
\hline Edad & Valor $\mathbf{t}$ & Valor $\mathbf{P}$ \\
\hline 8 & $-0,92$ & 0,375 \\
9 & $-0,3$ & 0,0042 \\
10 & 0,338 & 0,738 \\
11 & 5,14 & 0,0001 \\
12 & 5,33 & 0,000 \\
13 & 8,20 & 0,000 \\
14 & 12,81 & 0,000 \\
15 & 18,97 & 0,000 \\
\hline
\end{tabular}

Tabla VI. Comparación de estadios de Análisis CVM de Baccetti y Análisis de Lamparski en grupos de edad en Mujeres.

\begin{tabular}{lcc}
\hline Edad & Valor t & Valor $\mathbf{P}$ \\
\hline 8 & $-0,51$ & 0,002 \\
9 & 3,4 & 0,003 \\
10 & 4,37 & 0,003 \\
11 & 6,49 & 0,000 \\
12 & 10,40 & 0,000 \\
13 & 10,60 & 0,000 \\
14 & 14,29 & 0,000 \\
15 & 15,69 & 0,000 \\
\hline
\end{tabular}

Los grupos de población masculina de 11 a 15 años presentaron una diferencia significativa siendo mayor los valores para el análisis de Lamparski. Los grupos de población femenina de 9 a 15 años de edad también presentaron diferencias significativas con un mayor valor en los datos obtenido para el análisis de Lamparski.

\section{DISCUSIÓN}

En estudios relacionados a la maduración ósea y en los cuales se obtuvieron resultados que coinciden con los del presente estudio está el realizado por Luna Mata et al. (2007), quienes en el Departamento de Estomatología del Hospital Infantil de México, realizaron un estudio para determinar si existe relación entre los estadios carpales por el método de Björk con los estadios de las vértebras cervicales según Lamparski, la muestra final fueron 51 mujeres y 49 hombres sus resultados fueron favorecedores en la aplicación del análisis de Lamparski como medio diagnóstico; estos último debido a la nula necesidad de exponer a mayor radiación a los pacientes y a que dicho análisis carpal no se adecua totalmente a las etapas de maduración en la población en la cual fue estudiado.
Lo mismo ocurre con el estudio de MoralesDomínguez et al. (2014), en el Departamento de Estomatología del Hospital Infantil de México "Dr. Federico Gómez" quienes realizaron el mismo estudio, donde la muestra total fue de 213 pacientes, 105 mujeres y 108 hombres, todos fueron pacientes sanos entre los 6 y 17 años de edad, obteniendo en general un $66,61 \%$ de correlación para el sexo femenino y para el sexo masculino coinciden el $57,3 \%$ de los casos.

Usando como método el análisis CVM de Baccetti, se encuentra el estudio de Portales Carbonel \& Portocarrero Reyes (2013), en una población peruana de 150 pacientes de 7 a 18 años, quien encontró que, en todos los estadios de maduración esquelética de las vértebras cervicales con el método de Hassel \& Farman así como con el método de Baccetti, el promedio de la maduración ósea fue menor lo que se ve reflejado también en la población del presente estudio y los datos que Ortiz et al. encontró en una población venezolana de 47 pacientes pretratamiento de 5 a 12 años de edad, donde el $57 \%$ de la población total se encontró en un CMVSI, mientras que el $43 \%$ se encontró en CMVSII, siendo el dato más relevante que a partir de los 10 años el 100 $\%$ de los pacientes y hasta los 12 años; se encontraron en CMVSII; la cual presenta los datos también similares a los de este estudio.

ALVARADO-TORRES, E.; GUTIÉRREZ-ROJO, J. F. \& ROJAS-GARCÍA, A. R. Comparison of bone maturation of cervical vertebrae using Baccetti and Lamparski methods in patients 8 to 15 years of age. Int. J. Odontostomat., 10(1):63-67, 2016.

ABSTRACT: Maturation can be defined as a gradual process over time, presenting successive qualitative changes in the anatomical and physiological organization during puberty to the adult status maturity. In the diagnosis and treatment in orthodontics it is important to know the stages of bone maturation. This can provide us relevant information that allows us to determine pace and time of maturation individual. The use and application techniques vary between and have a common goal, the classification of children and adolescents in stages of maturation. The objective of the study was to compare bone maturation of the cervical vertebrae using the methods of Baccetti and Lamparski in a population of Nayarit. The sample consisted of 298 lateral radiographs of the skull between ages of 8-15 years old. The maturity stages of the groups of CVM and Lamparski method, in men and women, were compared using Student $\mathrm{t}$ test. In the masculine population between 8 and 10 years significant differences were not found between both analysis; in the 8-year-old female group and the 9-year-old males, 
differences were found, with the CVM Baccetti analysis having higher values. In the other study groups, differences were also found showing higher values in the Lamparski analysis. The stages of maturation in the two types of analysis showed a marked difference in their interpretations. Lamparski analysis being more reliable as a diagnostic method for bone maturation.

KEY WORDS: bone maturation, vertebrae Lamparski, Baccetti.

\section{REFERENCIAS BIBLIOGRÁFICAS}

Baccetti, T.; Franchi, L. \& McNamara, J. A. Jr. The cervical vertebral maturation (CVM) method for the assessment of optimal treatment timing in dentofacial orthopedics. Semin. Orthod., 11(3):119-29, 2005.

Chen, F.; Terada, K. \& Hanada, K. A new method of predicting mandibular length increment on the basis of cervical vertebrae. Angle Orthod., 74(5):630-4, 2004.

Echarri, P. A. Diagnóstico en Ortodoncia. Estudio Multidisciplinario. Barcelona, Quintessence, 1998. pp.539.

Gandini, P.; Mancini, M. \& Andreani, F. A comparison of handwrist bone and cervical vertebral analyses in measuring skeletal maturation. Angle Orthod., 76(6):984-9, 2006.

Gómez-Campos, R.; de Arruda, M.; Hobold, E.; Abella, C. P.; Camargo, C.; Martínez Salazar, C. \& Cossio-Bolaños, M. A. Valoración de la maduración biológica: usos y aplicaciones en el ámbito escolar. Rev. Andal. Med. Deporte, 6(4):151-60, 2013.

Luna Mata, A. D.; Martínez López, C.; González Ramírez, E.; Canseco Jiménez, J.; Cuairán Ruidíaz, V. \& Gaitán Cepeda, L. A. Relación existente entre el tamaño y forma de las vértebras cervicales con los estadios de maduración ósea carpal. Rev. Odontol. Mex., 11(3):121-8, 2007.

Mejía-Garduño, G.; Canseco-Jiménez, J.; Martínez-López, C.; Reyes-López, A. \& Cuairán-Ruidíaz, V. Correlación entre los estadios de maduración carpal y los estadios de desarrollo del canino mandibular en pacientes que reciben tratamiento ortodóncico. Rev. Odontol. Mex., 18(1):9-13, 2014.

Morales-Domínguez, E. I.; Martínez-López, C.; GonzálezRamírez, E.; Canseco-Jiménez, J. \& Cuairán Ruidíaz, V. Relación existente entre el tamaño y forma de las vértebras cervicales con los estadios de maduración ósea carpal. Parte II. Parte II. Rev. Mex. Ortod., 2(1):2431, 2014.
Ortiz, M.; Godoy, S.; Fuenmayor, D.; Farias, M.; Quirós, O.; Rondón, S. \& Lerner, H. Método de maduración ósea de las vertebras cervicales, en pacientes del Diplomado de Ortodoncia Interceptiva, UGAMA - 2006. Rev. Latinoam. Ortodon. Odontopediatr., 2007. Disponible en: http:// www.ortodoncia.ws/publicaciones/2007/ maduracion_osea_vertebras_cervicales.asp

Perinetti, G.; Caprioglio, A. \& Contardo, L. Visual assessment of the cervical vertebral maturation stages: A study of diagnostic accuracy and repeatability. Angle Orthod., 84(6):951-6, 2014.

Portales Carbonel, C. X. \& Portocarrero Reyes, W. Edad promedio de aparición de los estadios de maduración esquelética de las vértebras cervicales con el Método de Hassel y Farman y Baccetti. Rev. Dent. Chile, 104(3):19-23, 2013.

San-Román, P.; Palma, J. C.; Oteo, M. D. \& Nevado, E. Skeletal maturation determined by cervical vertebrae development. Eur. J. Orthod., 24(3):303-11, 2002.

Dirección para Correspondencia:

Emerik Alvarado Torres

Especialidad en Ortodoncia

Universidad Autónoma de Nayarit.

Nayarit

MÉXICO

Emai: emerik_alvaradotorres@hotmail.com

Recibido : 01-10-2015

Aceptado: 19-01-2016 